\title{
DEVICE FOR CONTROL OF HUMIDITY OF LOOSE MATERIALS IN THE STREAM OF THE SUBSTANCE
}

Alexander IVASHINA, Department of Electrical Supply and Operation of Electrical Equipment, Stavropol State Agrarian University, Stavropol, 12 Zootechnicheskiy Ln, Russia; av_ivashina49@mail.ru

Vitaly GRINCHENKO, Department of Electricity in agriculture, Stavropol State Agrarian University, Stavropol, 12 Zootechnicheskiy Ln, Russia; grinchen_ko@mail.ru (corresponding author)

Valery ZHDANOV, Department of Electrical Supply and Operation of Electrical Equipment, Stavropol State Agrarian University, Stavropol, 12 Zootechnicheskiy Ln, Russia,

Svetlana LYUBAYA, Department of Physics, Stavropol State Agrarian University, Stavropol, 12 Zootechnicheskiy Ln, Russia; unil.sgau@ya.ru

\begin{abstract}
Improvement of the design of the sample preparation device for bulk materials during the flow control of their moisture content, based on the use of the vibrating process to stabilize the bulk density, will ensure continuity of the flow of bulk materials. The accuracy of humidity measurement will be increased by using dielectric metric methods due to the decrease in the through conductivity of loose materials, which is associated with the rupture of contact bridges between their particles in the vibrating mass. An analysis of the results of an experimental study of bulk materials in a vibrating state shows that the use of such a grain state is advisable for the following reasons. Firstly, at a constant vibration amplitude, the bulk density of the grain mass stabilizes, and secondly, it's through conductivity sharply decreases, which is explained by the ruptures of contact bridges between the particles in the vibrating fluid mass of bulk material. Combination of the process of vibration for the purpose of stabilizing the bulk density of bulk material with its simultaneous transportation through the cavity of the vibration chamber is carried out in the device proposed by the authors. In order to ensure the stabilization of the bulk density of bulk material, a device has been new developed.
\end{abstract}

Keywords: bulk material, density, grain, hopper, moisture, primary converter.

\section{INTRODUCTION}

In modern moisture control devices for bulk materials, reliable and reproducible results cannot be obtained without special preparation of the sample. Preparation of the sample is reduced to maintaining its constant density throughout the range of controlled humidity and is one of the most important tasks of sample preparation for moisture control devices used in flows of various bulk materials.

In general, the function of sample preparation for the control of the moisture content of bulk materials is reduced to solving the following problems: a) stabilization of bulk density throughout the moisture range; b) averaging the material with respect to moisture and composition, both along the axis of motion and in a direction perpendicular to it; c) the formation of a measuring zone in the direction of the axis of flow.

Problem b) is not always possible to solve in the devices for controlling the moisture content of bulk materials, since bulk materials are delivered in lots with different humidity. Problem c) is not difficult to solve, so let us consider the solution of problem a).

A relatively simple and acceptable way of stabilizing the density of a sample of bulk material, as an interfering factor, is its compulsory compaction, or a spill with a free gravitational flow (Kok Y. Y. et al, 2013). There are devices for preparing a sample with a free (gravitational) flow of material (Fig. 1). The test results of such devices have shown that they can be recommended for stationary flows of grain and granular materials, but are of little use for finely dispersed materials, for example, flour prone to stick to electrodes (Azadi M.S., Younesi E., 2013). The flow devices have an excessive number of electrodes that impair the operation of the primary converters, since the smoothness of the renewal of the material is disturbed, in addition the fasteners are placed in the cavity of the sample preparation device and disturb the smooth flow of the grain mass, forming floating voids (Khairetdinova A. F. et al, 2012). 


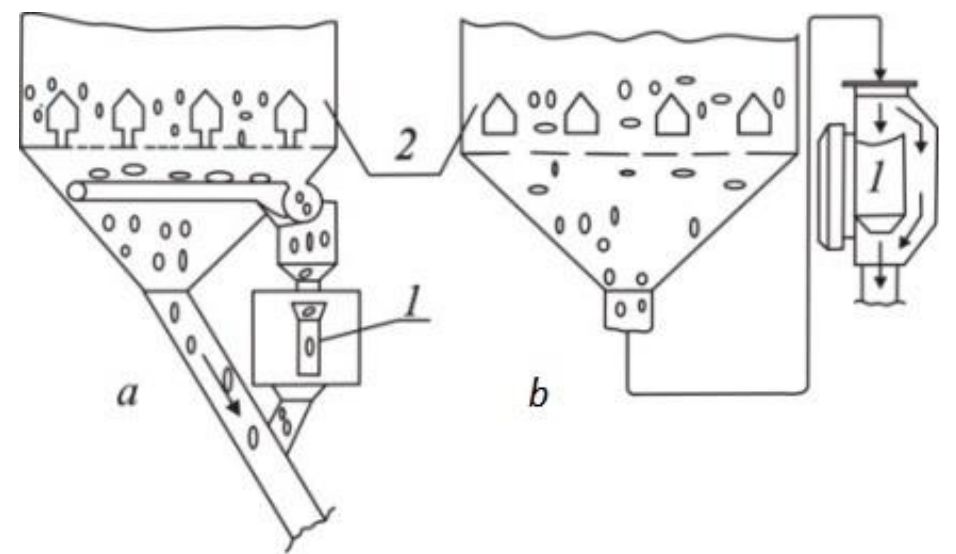

Figure 1. Schemes for placement of flow-through devices for sample preparation with gravitational flow of grain to control its moisture content in silo grain dryers: a - in experimental automatic humidity control systems; $b$ - in domestic flow hygrometers; 1 - the primary converter; 2 - grain dryer

One of the main disadvantages of flow devices with gravitational flow of loose materials is zeroing of the moisture indicator during short breaks or significant unevenness in the supply of bulk materials, which leads to malfunctions in the operation of automatic humidity controllers and complicates the operation of the operating personnel of grain dryers.

The sample preparation device with a vibration seal of loose material is shown in Fig. 2. It consists of a receiving hopper 3, a chamber 2 in the wall of which there is a window 1, for the flow of loose material, and electrodes 4 isolated from the metal walls of the chamber.

Moisture meter can be used to measure humidity during drying in carousel, mine type dryers and others when harvesting and processing cereals at enterprises of the agro-industrial complex and food industry

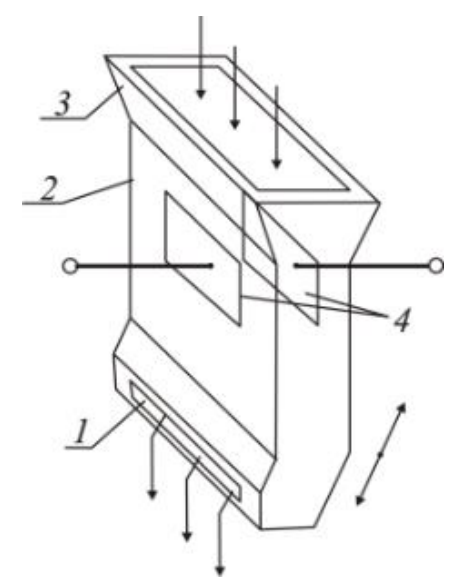

Figure 2. Sample preparation unit with vibration seal:

1 - the window; 2 - the chamber; 3 - hopper bunker; 4 - electrodes of the primary converter

Finely dispersed bulk materials with a polydisperse granulometric composition are divided into size classes and are subject to significant adhesion during vibratory compaction. The flow of material through the cavity of such a measure becomes unstable with a width of more than $3 \mathrm{~cm}$.

These factors impose significant restrictions on the use of such devices. However, such devices are used for materials of monodispersed composition, for example, granulated mineral fertilizers, grains, etc. Electrodes 4 of the capacitive humidity transmitter are mounted in the chamber flush with the internal surfaces of the walls.

Sample preparation devices, bulk with a power seal, are divided into discontinuous devices, screw and roller devices. Fig. 3 shows a bulk device with a screw feeder.

The bulk material from the hopper 1 flows along the vertical section of the pipeline into the zone of the capacitive humidity primary transducer 3 and is removed by the screw 4 . The excess material from the hopper 1 passes through the bypass pipe 2 into the main pipeline.

The disadvantages of such a device include the need to use a rotating electric drive. Short interruptions in the supply of bulk materials lead to the reset of the indicator of the measuring transducer.

The screw-type sample-preparation devices combine the function of displacement and compaction and are structurally made in the form of a short screw that contains several turns. 


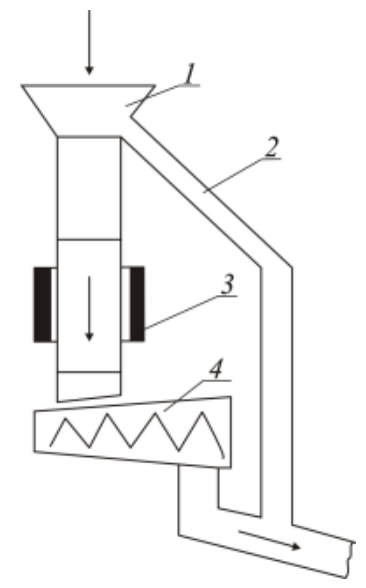

Figure 3. The device for preparing a sample of bulk materials with an auger feeder:

1 - bunker; 2 - bypass pipe; 3 - electrodes of the capacitive primary converter; 4 -auger

Feed sampler (not shown in Fig. 4) is loaded into the receiving hopper 1, from which it is taken by the feeder screw 2. Under the action of it and the forming nozzle 3, the mass enters the guide pipe 4 and the packing nozzle 6 . Due to friction of their walls, the food is compacted. The capacitive primary transducer 5 of the single-sided field has alternating high and low potential rings-electrodes located inside the pipe 4 of insulating material.

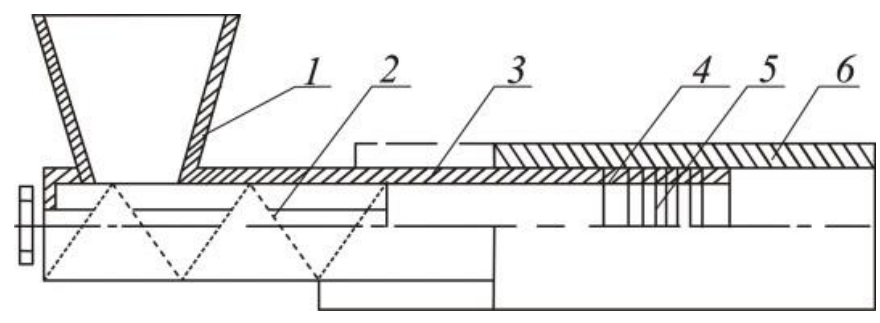

Figure 4. Sample preparation device with screw feeder:

1 - receiving hopper; 2 - auger feeder; 3, 6 - forming and sealing nozzles; 4 - a directing pipe; 5 - capacitive primary converter

Humidity is determined by means of an electrical circuit with parametric modulation. The specified bulk density of feed in the sensor is maintained by means of a special system. For this purpose, through holes in the sealing packing 6 are drilled along the circumference, strain gauges are installed between them. When the volume density of the feed changes, its pressure on the walls of the packing nozzle changes and a signal appears in the strain gauges, which controls the actuator moving the nozzle.

The device is difficult in design, has a rotating electric drive and is practically unsuitable for monitoring the moisture content of the grain, as it will lead to its injury.

Sliding sample preparation machines with a power seal create the required sealing effect by its own mass or additional weighed loads. In such devices, typically, the coplanar capacitive primary converters are used. Constant contact with the abrasive medium leads to intensive wear of the primary transducer, and as a consequence to a change in its capacitance. This disadvantage is eliminated by selecting a pair of dielectric materials - electrodes, using a hard alloyceramic pair. Such devices have not found application in the measurement of grain moisture due to their high metal content, the need for additional averaging units and equalizing the flow of bulk material.

Slip devices for preparation of a sample with self-packing include sublent (non-contact) devices. The electrodes of the primary converter are pressed against the transport belt (Fig. 5, a).
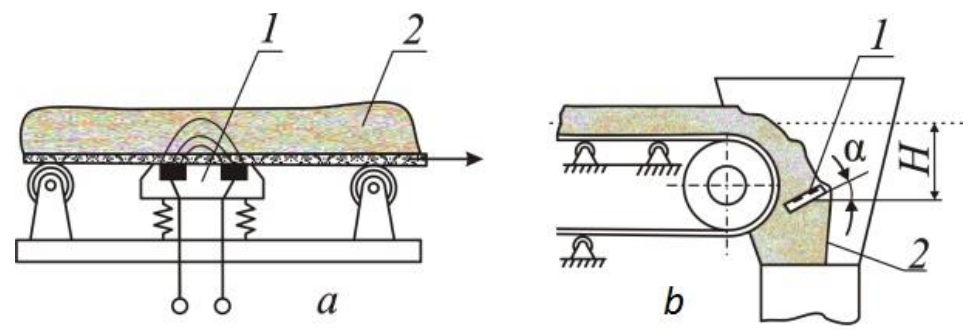

Figure 5. Sampling devices with self-sealing: a) sliding under-tap primary converter; b) a primary transducer installed in a hopper; 1 - the primary converter; 2 - the bulk material 
The geometric dimensions of such primary converters are chosen in such a way that the electric field covers both the tape and the layer of bulk materials located on the tape. Such devices are used for chemically aggressive materials or with high active losses and are not suitable for cases of sharp uneven performance.

To the same group of preparation devices, the samples include those that are installed in overflowing streams, bunkers, etc. The primary converter is fixed and turned by its working plane at the angle of the symbol to the horizon. This angle is chosen so as to ensure a good glide of bulk materials along the working plane of the primary converter. The reproducibility of the results of moisture control of bulk materials is achieved by selecting the height $\mathrm{H}$ of the incident flow (Fig. 5, b).

The disadvantages of such devices include the possibility of their use only for technological purposes, when a strict continuity of the incident flow is ensured, its permanence there is no migration of the flow along the section of the estuary.

Improvement of the design of the sample preparation device for bulk materials during the flow control of their moisture content, based on the use of the vibrating process to stabilize the bulk density, will ensure continuity of the flow of bulk materials. The accuracy of humidity measurement will be increased by using dielectric metric methods due to the decrease in the through conductivity of loose materials, which is associated with the rupture of contact bridges between their particles in the vibrating mass.

\section{MATERIAL AND METHODS}

An analysis of the results of an experimental study of bulk materials in a vibrating state shows that the use of such a grain state is advisable for the following reasons. Firstly, at a constant vibration amplitude, the bulk density of the grain mass stabilizes, and secondly, it's through conductivity sharply decreases, which is explained by the ruptures of contact bridges between the particles in the vibrating fluid mass of bulk material.

The purpose of the study is to combine the process of vibration dampening to stabilize bulk density of bulk material and its simultaneous transportation through a vibration chamber. To ensure the stabilization of bulk density of bulk material, the authors developed a device, the basic diagram of which is shown in Fig. 6.

The device comprises a receiving hopper 1, a U-shaped flow chamber 2 with different knee lengths mounted vertically and fixed to the elastic members 3 . The electromagnetic vibrators 4 and 5 are connected by means of a yoke clamp 6 with a humidity sensor 7 on the short knee.

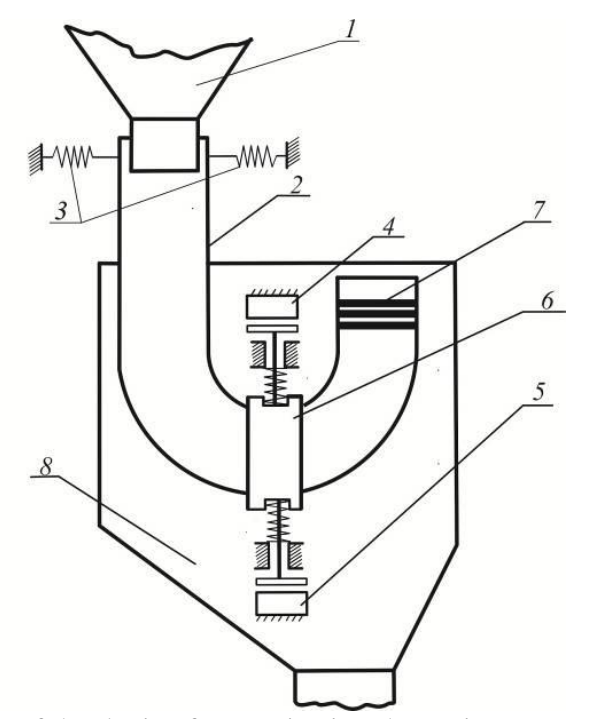

Figure 6. Schematic diagram of the device for monitoring the moisture content of bulk materials:

1 - receiving hopper; 2 - vibration chamber; 3 - elastic elements; 4, 5 - electromagnetic vibrators; 6 - a collar; 7 - sensor; 8 - prefabricated hopper

The humidity sensor (capacitive type) can be made of several coaxial sections. At the output of the vibrating chamber, a prefabricated hopper 8 is installed.

The device works as follows. Bulk material through the receiving hopper 1 enters U-shaped vibration chamber 2, under the influence of vibration, it is "flowing" through a continuous flow through the cavity of the U-shaped chamber, and therefore through the cavity of the sensor 7 connected to the secondary device (not shown in the figure) due to the difference in pressure due to the level difference), which measures the moisture content of the bulk material, the latter then enters the collecting hopper 8 . The measuring circuit of the device can have various versions used in dielectric metric hydrometers.

Electromagnetic vibrators 4 and 5 create forced harmonic oscillations with the frequency of the supplying alternating voltage, while the vibrators 4 and 5 are affected by the positive (negative) and negative (positive) half-periods of the voltage applied to them, respectively. The use of two vibrators makes it possible to avoid tuning the vibrating chamber into resonance with the frequency of natural oscillations. The amplitude of the oscillations determines the traveling speed (flow rate) of the bulk material and is controlled by the appropriate measurement of the supply voltage of the coil of the electromagnetic vibrator. 
Let us derive the main relations between the geometric dimensions of the U-shaped chamber and the coefficient of friction of the bulk material on its inner surface.

We assume the assumptions of the equality of the internal diameter of the chamber $\mathrm{d} 1$, and the diameter $\mathrm{d} 2 \mathrm{of}$ the tube bending (Fig. 7), at which equalities.

$$
l_{s l 1} \approx l_{1} ; l_{s l 2} \approx l_{2},
$$

where $l_{s l l}$ and $l_{s l 2}$ - the lengths of the midline of the long and short bends.

Let's divide conditionally the grain mass in the flow chamber into two parts. Masses of loose material in knees of length $l_{s l l}$ and $l_{s l 2}$ are equal to $m_{1}$ and $m_{2}$, respectively, the U-shaped chamber performs oscillatory movements in the vertical plane. We write the equation of dynamics for moving masses, using the d'Alembert principle:

$$
m_{1}(g \pm a)-m_{2}(g \pm a)=C_{f r} \cdot N=C_{f r}\left(N_{1}+N_{2}\right)
$$

where $\quad \mathrm{g}-$ the acceleration of gravity;

$a$ - vibration acceleration;

$C_{f r}$-the coefficient of friction of the material under study against the wall of the tube;

$N_{l}, N_{2}$ - the normal force of pressure on the internal walls of the knees of length $l_{l}$ and $l_{2}$, respectively.

The normal force of pressure in the knee is $l_{l}$, (Fig. 7):

$$
N_{1}=\gamma(g \pm a) \cdot \pi d_{1} \int_{0}^{e} x d x=\gamma(g \pm a) \cdot \frac{\pi d_{1} \cdot l_{1}^{2}}{2},
$$

where $\quad \gamma$-the density of bulk material; $d_{l}$ is the tube diameter; $x$ and $d x$ - the current coordinate and its increment, respectively.

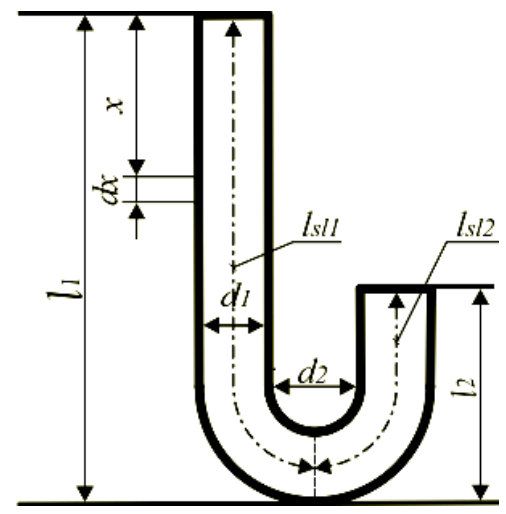

Figure 7. Scheme for choosing the ratios of the geometric dimensions of a vibratory flow chamber

Similarly, the pressure force in the knee of length $l_{2}$ :

$$
N_{2}=\gamma(g \pm a) \frac{\pi d_{1} \cdot l_{2}^{2}}{2}
$$

The masses in the knees of length $l_{1}$ и $l_{2}$ are determined as follows:

$$
m_{1}=\frac{\pi \cdot d_{1}^{2} \cdot l_{1} \cdot \gamma}{4}, m_{2}=\frac{\pi \cdot d_{1}^{2} \cdot l_{2} \cdot \gamma}{4} .
$$

Substituting the values of $N_{l}, N_{2}, m_{l}$ and $m_{2}$ into expression (1), we finally obtain

$$
\begin{gathered}
\frac{1}{2} \beta(1-\alpha)=K_{m p}\left(1+\alpha^{2}\right) \text { or } \frac{1}{2} \beta \frac{(1-\alpha)}{\left(1+\alpha^{2}\right)}=K_{m p} \\
\text { where } \quad \beta=\frac{d_{1}}{l_{1}} ; \quad \alpha=\frac{l_{2}}{l_{1}} .
\end{gathered}
$$


In the general case, the condition for the possibility of moving material in the tube is inequality

$$
\frac{1}{2} \beta \frac{(1-\alpha)}{\left(1+\alpha^{2}\right)} \geq K_{k f} \text {. }
$$

\section{RESULTS AND DISCUSSION}

From the analysis of expression (3) it follows that the left-hand side of the inequality increases with increasing tube diameter and constant value of the lengths of the knees. If the value of $\beta$ is the same as the value of $\alpha$ decreases, the left-hand side of the inequality also increases, that is, the conditions for the movement of the material are improved.

The values of friction coefficients of bulk materials are determined experimentally.

The developed device can be used to control and regulate humidity during the production of flour, sugar, milk powder, fish meal, grain of various crops, starch, dry yeast, cocoa, gelatin, cereals.

Experimental studies have established that the developed design of the vibrating chamber ensures stabilization of the bulk density of bulk materials in the flow.

The through conductivity of such materials is sharply reduced, which is explained by the rupture of contact bridges in the fluidized bulk of loose materials.

Tests of cameras with a diameter of $50 \mathrm{~mm}$ and $100 \mathrm{~mm}$ and lengths of short and long elbows $150 \mathrm{~mm}$ and $350 \mathrm{~mm}$, respectively, showed that the flow of bulk material passing through the capacitor sensor is stable.

Short interruptions in the flow of bulk materials do not lead to a periodic zeroing of the moisture indicator. With such interruptions, the level of bulk material in the long knee becomes equal to its level in the short knee. Therefore, such interruptions cannot be perceived incorrectly by the service personnel and cause erroneous actions, and also will not cause significant errors when measuring the moisture content of bulk material.

Further researches of the authors are planned to be carried out using the results obtained in the direction of simplifying the design of the vibrating chamber, examining the electrical characteristics of various bulk materials in the vibrating fluid state, and improving measurement methods.

\section{CONCLUSIONS}

1. The application of the vibrating process for the transport of bulk materials has made it possible to develop a design of a vibration-proof device for monitoring the moisture content of bulk materials in the form of a U-shaped chamber.

2. The device ensures the stabilization of bulk density of loose materials in the flow.

3. The use of two vibrators allows you to abandon the tuning of the vibrating chamber into resonance with the frequency of natural oscillations. The amplitude of the oscillations determines the speed of movement (consumption) of the grain mass and is regulated by the appropriate measurement of the supply voltage of the coil of the electromagnetic vibrator.

4. The decrease in the through conductivity of loose materials in the vibrating state is explained by the breakage of contact bridges between the particles of bulk materials. This makes it possible to increase the accuracy of measurements with the use of dielectric measurement methods for humidity.

\section{REFERENCES}

1. Azadi, M.S., Younesi, E. 2013. The effects of storage on germination characteristics and enzyme activity of sorghum seeds. Journal of Stress Physiology \& Biochemistry, Vol. 9(4), pp. 289-298.

2. Khairetdinova, A.F., Saitov, R.I., Abdeev, R.G., Talipov, N.S. 2012. A study of a hygrothermal method of grain moisture control in the drying process. Measurement techniques, Vol. 55(1), pp. 104-107. https://doi.org/10.1007/s11018-012-9924-z

3. Kok Y. Y., Hou K. M., Li L. Y., Jamaliah S., Zulkifly A. 2013. A Small and Slim Coaxial Probe for Single Rice Grain Moisture Sensing. Sensors, Vol. 13(3), pp. 3652-3663.

4. Vostrukhin, A., Vakhtina, E. 2015. Analog-to-digital Converter based on the transient process in RC-circuit. Proceedings of the 14th International Scientific Conference "Engineering for Rural Development", vol. 14, pp. 452-457, Latvia University of Agriculture, Jelgava, Latvia, 20-22 May 2015. 